\title{
An inguinal hernia sac tumor of extrahepatic cholangiocarcinoma origin
}

\author{
Naoyuki Yokoyama*1, Yoshio Shirai ${ }^{1}$, Hidehiro Yamazaki ${ }^{2}$ and \\ Katsuyoshi Hatakeyama ${ }^{1}$
}

\author{
Address: ${ }^{1}$ Division of Digestive and General Surgery, Niigata University Graduate School of Medical and Dental Sciences, Asahimachi-dori 1-757, \\ 951-8510, Niigata, Japan and 2Department of Surgery, Niigata Minami Hospital, Meike-Shinmei 1-7-1, 950-8601, Niigata, Japan \\ Email: Naoyuki Yokoyama* -yokonao@med.niigata-u.ac.jp; Yoshio Shirai - shiray@med.niigata-u.ac.jp; \\ Hidehiro Yamazaki - hyamazaki@niigataminami-hp.com; Katsuyoshi Hatakeyama - khatake@med.niigata-u.ac.jp \\ * Corresponding author
}

Published: 06 March 2006

World Journal of Surgical Oncology2006, 4:13 doi:10.1186/1477-7819-4-13

This article is available from: http://www.wjso.com/content/4/I//3

(c) 2006Yokoyama et al; licensee BioMed Central Ltd.

This is an Open Access article distributed under the terms of the Creative Commons Attribution License (http://creativecommons.org/licenses/by/2.0), which permits unrestricted use, distribution, and reproduction in any medium, provided the original work is properly cited.
Received: 02 November 2005

Accepted: 06 March 2006

\begin{abstract}
Background: Metastatic hernia sac tumor from biliary malignancy is extremely rare with only one such case previously reported. We herein report an additional case of extrahepatic cholangiocarcinoma presenting as a hernia sac tumor.

Case presentation: A 78-year-old man presented with an irreducible right inguinal hernia associated with a firm tumor, $2.0 \mathrm{~cm}$ in diameter. A computed tomography scan demonstrated a soft tissue density mass with heterogeneous enhancement within the right inguinal canal. The patient underwent a hernia repair and the hernia sac tumor was resected. Histological examination of the tumor revealed a metastatic adenocarcinoma suggesting the tumor was of pancreato-biliary origin. Further investigation using imaging studies disclosed a primary tumor in the upper bile duct. The patient died of the disease nine months after the resection.

Conclusion: Hernia sac tumors should be considered when an irreducible, growing mass appears within an inguinal hernia. Computed tomography may be useful for the early detection of hernia sac tumors from undiagnosed intra-abdominal malignancies.
\end{abstract}

\section{Background}

Tumors associated with inguinal hernias are rare, occurring in less than $0.5 \%$ of surgically excised sacs [1]. Thus far, computed tomography (CT) features of hernia sac tumors have been poorly documented [2,3]. We herein report the case of a hernia sac tumor that originated from an extrahepatic cholangiocarcinoma, and specifically focus on the CT features exhibited by a metastatic tumor within an inguinal hernia.

\section{Case presentation}

A 78-year-old man presented with a tender mass in the right groin that had appeared five months earlier and had gradually grown. He had a three-year history of right inguinal hernia. Physical examination revealed an irreducible right inguinal hernia associated with a firm tumor, $2.0 \mathrm{~cm}$ in diameter. A plain CT scan demonstrated a soft tissue density mass within the right inguinal canal, which had no connection with intra-abdominal organs (Figure 1) and showed heterogeneous enhancement on a contrast CT scan (Figure 2). After being diagnosed with a 


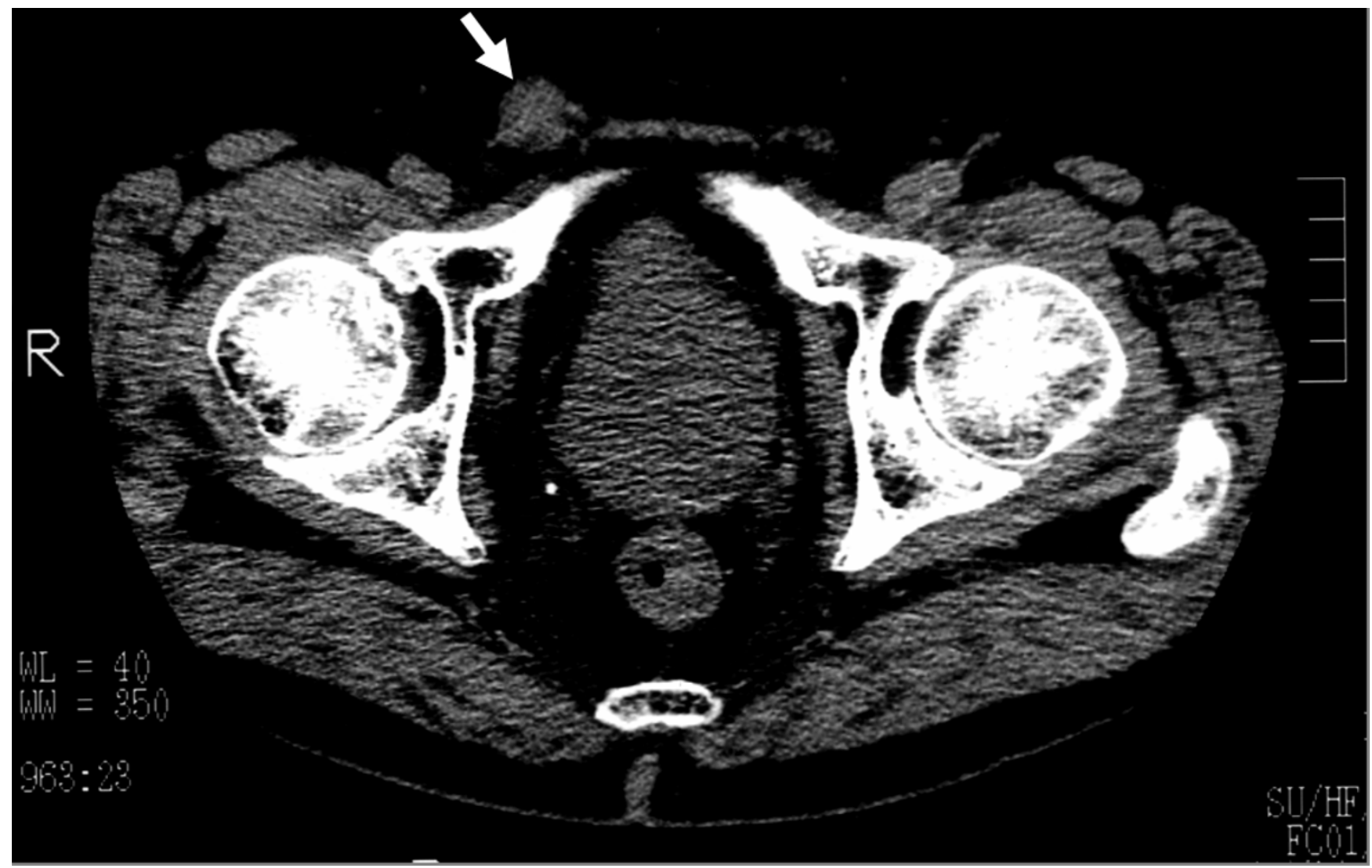

Figure I

Plain computed tomography image showing a soft tissue density mass (arrow) within the right inguinal canal. The mass had no connection with intra-abdominal organs.

hernia sac tumor of unknown origin, the patient underwent a hernia repair (iliopubic tract repair) under spinal anesthesia. A white tumor located at the tip of the sac encompassed the testicular artery and vein, and was resected together with the sac and the testicular vessels. Histological examination of the tumor revealed a metastatic, moderately differentiated adenocarcinoma with perineural invasion, suggesting that the tumor was of pancreato-biliary origin. Further investigation using abdominal CT scans and ultrasonography disclosed a tumor in the upper bile duct associated with gallbladder swelling. The primary tumor was diagnosed as an extrahepatic cholangiocarcinoma. Further investigations detected no malignancies in other organs. The patient died of the disease nine months after the operation despite systemic chemotherapy using gemcitabine. At the time of his death, neither hernia recurrence nor local tumor relapse were found in the right inguinal region.

\section{Discussion}

Hernia sac tumors can be classified into one of three groups: intrasaccular, in which an organ bearing the tumor is incarcerated within the sac; saccular, in which the tumor encompasses the sac or spermatic cord structures; or extrasaccular, in which the tumor protrudes through the hernia defect but is located outside the sac [1]. Accordingly, the metastatic tumor described in this case can be classified as a saccular hernia sac tumor. The most common cause of saccular tumors is metastases from abdominal malignancies that migrate under the influence of gravity [1]. Hernia sac tumors are associated with a wide spectrum of tumor types. Among them, the most frequent primary site is the colon followed by the ovaries, prostate, pancreas, and appendix [1,4]. The hernia sac tumor described in this case originated from an extrahepatic cholangiocarcinoma, which is an extremely rare event with only one such case previously reported [4].

CT features of metastatic inguinal saccular tumors have been poorly documented, and to our knowledge there are only two documented cases that present CT findings of saccular tumors $[2,3]$. Yokota and colleagues reported that a saccular tumor of colonic carcinoma origin was depicted in a contrast CT scan as a soft tissue density mass with het- 


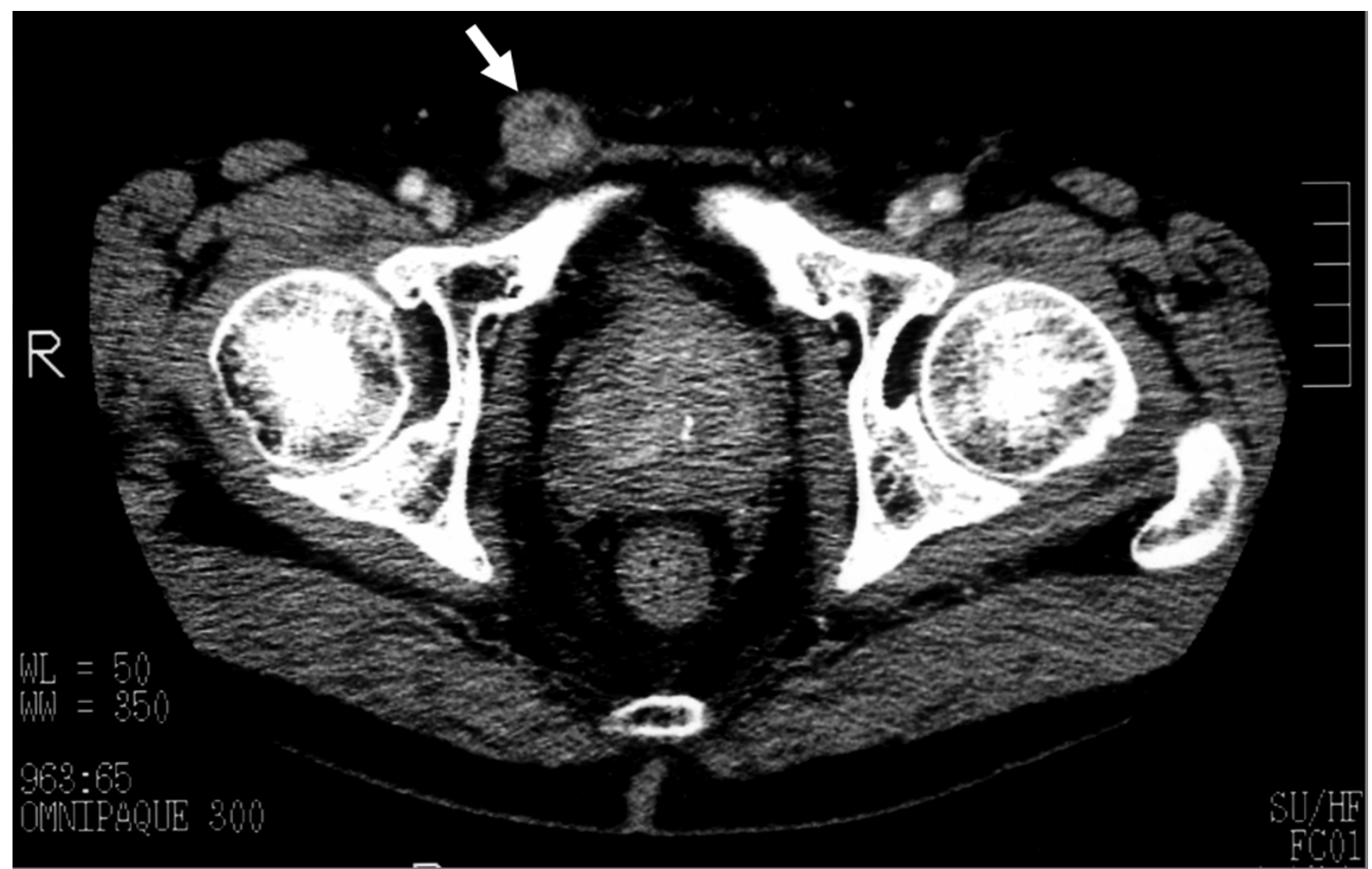

Figure 2

Contrast computed tomography scan image showing the inguinal tumor with heterogeneous enhancement (arrow).

erogeneous enhancement [2]. These CT scan findings are consistent with those described in the present case, suggesting that a soft tissue density mass with heterogeneous enhancement is a common CT feature of saccular tumors of gastrointestinal carcinoma origin. However, the contrast CT scan results for a saccular tumor originating from pseudomyxoma peritonei show a low-density mass without enhancement, suggesting a gelatinous composition [3]. CT scan findings from the present and previous studies highlight the potential use of preoperative CT scans for the differential diagnosis of hernia sac tumors.

\section{Conclusion}

Hernia sac tumors can be suspected when an irreducible, growing mass appears within an inguinal hernia. Since hernia repair offers an opportunity for peritoneal biopsy, selective microscopic examination of grossly abnormal hernia sac specimens is recommended. Preoperative CT imaging may also be useful for the early detection of her- nia sac tumors from undiagnosed intra-abdominal malignancies.

\section{Competing interests}

The author(s) declare that they have no competing interests.

\section{Authors' contributions}

NY: participated in the operation, searched the literature, and wrote the original manuscript.

YS: assisted in literature search and preparing the manuscript.

HY: participated in the operation and performed chemotherapy to the patients.

KH: supervised preparation of the manuscript and edited the final version for publication. 
All authors read and approved the final manuscript.

\section{Acknowledgements}

Written consent was obtained from the patient for reporting the case.

\section{References}

I. Nicholson CP, Donohue JH, Thompson GB, Lewis JE: A study of metastatic cancer found during inguinal hernia repair. Cancer 1992, 69:3008-30II.

2. Yokota R, Hata Y, Matsuoka S, Nakajima S, Makita K, Sano F: A case of sigmoid colon carcinoma metastasized to an inguinal hernia sac. J Japan Surg Asso 2003, 64:999-1002.

3. Shimoyama S, Kuramoto S, Kawahara M, Yamasaki K, Endo $H$, Murakami T, Kaminishi M: A rare case of pseudomyxoma peritonei presenting an unusual inguinal hernia and splenic metastasis. J Gastroenterol Hepatol 200 I, 16:825-829.

4. Lowenfels AB, Rohman M, Ahmed N, Lefkowitz M: Hernia-sac cancer. Lancet 1969, I:65I.

Publish with Bio Med Central and every scientist can read your work free of charge

"BioMed Central will be the most significant development for disseminating the results of biomedical research in our lifetime. "

Sir Paul Nurse, Cancer Research UK

Your research papers will be:

- available free of charge to the entire biomedical community

- peer reviewed and published immediately upon acceptance

- cited in PubMed and archived on PubMed Central

- yours - you keep the copyright

Submit your manuscript here:

http://www.biomedcentral.com/info/publishing_adv.asp 